\title{
Rapid and recent allopatric speciation in firs from central Mexico: evidence for reinforcement?
}

\author{
Gustavo Giles-Pérez ${ }^{1}$, Erika Aguirre-Planter ${ }^{1}$, Luis Eguiarte ${ }^{1}$, and Juan Jaramillo-Correa ${ }^{1}$ \\ ${ }^{1}$ Instituto de Ecología, Universidad Nacional Autónoma de México
}

May 10, 2021

\begin{abstract}
Secondary contact of species that have evolved partial reproductive isolation in allopatry may result in several outcomes, which range from rampant hybridization to barrier reinforcement. Reinforcement arises from reduced hybrid fitness, which promotes assortative mating and hence speciation. In plants, self-fertilization and disjunctions in reproductive-phenology are often invoked as evidence of reinforcement. However, local adaptation and pleiotropic effects during colonization can also lead to reproductive isolation without reinforcement. We explored these possibilities in a fir species complex (Abies flinckii - A. religiosa) distributed in 'sky-islands' along the Trans-Mexican Volcanic Belt (TMVB), in central Mexico. Despite co-occurring in two independent sympatric regions (west and center), these two taxa seem to rarely interbreed because of disjunct reproductive phenologies. We genotyped 1,147 SNPs, generated by GBS across 23 populations, and compared multiple demographic scenarios, built based on the geological history of the TMVB. The best-fitting model suggested a recent species split (for a conifer), dating back to $\sim 1.2 \mathrm{Ma}$, together with early asymmetric gene flow (mostly from A. flinckii into A. religiosa), limited to the central sympatric region. Coupled with the lack of support for colonization models, the summary statistics (f, Hobs, FST, $\vartheta \pi$, etc.) and historical demographic inferences made herein point to a rapid speciation with an early development of reinforcement, as a putative mechanism for avoiding hybridization. The role of reinforcement should be thus further explored in the (sub)tropics, as likely explanation for how species diversity is generated and maintained.
\end{abstract}

\section{Hosted file}

Main_document.pdf available at https://authorea.com/users/412887/articles/521401-rapid-andrecent-allopatric-speciation-in-firs-from-central-mexico-evidence-for-reinforcement 